\title{
Using epidemiological models to estimate the health effects of diet behaviour change: the example of tailored fruit and vegetable promotion
}

\author{
$J_{\text {Lennert Veerman }}{ }^{1}$, Jan J Barendregt $^{1,2}$, Johan P Mackenbach ${ }^{1}$ and Johannes Brug ${ }^{1}$ \\ 'Department of Public Health, Erasmus MC - University Medical Centre Rotterdam, PO Box 1738, 3000 DR \\ Rotterdam, The Netherlands: ${ }^{2}$ School of Population Health, University of Queensland, Brisbane, Queensland, \\ Australia
}

Submitted 5 January 2005: Accepted 31 August 2005

\begin{abstract}
Objective: To explore the use of epidemiological modelling for the estimation of health effects of behaviour change interventions, using the example of computertailored nutrition education aimed at fruit and vegetable consumption in The Netherlands.

Design: The effects of the intervention on changes in consumption were obtained from an earlier evaluation study. The effect on health outcomes was estimated using an epidemiological multi-state life table model. Input data for the model consisted of relative risk estimates for cardiovascular disease and cancers, data on disease occurrence and mortality, and survey data on the consumption of fruits and vegetables.

Results: If the computer-tailored nutrition education reached the entire adult population and the effects were sustained, it could result in a mortality decrease of 0.4 to $0.7 \%$ and save 72 to 115 life-years per 100000 persons aged 25 years or older. Healthy life expectancy is estimated to increase by 32.7 days for men and 25.3 days for women. The true effect is likely to lie between this theoretical maximum and zero effect, depending mostly on durability of behaviour change and reach of the intervention.

Conclusion: Epidemiological models can be used to estimate the health impact of health promotion interventions.
\end{abstract}

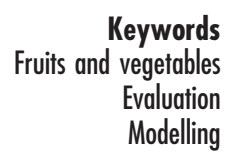

Health promotion interventions are intended to improve health. Good practice requires that their effects be evaluated. Typically, health promotion interventions aim to promote health behaviours, i.e. behaviours that reduce the risk of disease, such as non-smoking, physical activity and fruit and vegetable intake. The degree to which this is achieved is assessed in impact evaluation. However, ultimately we are interested in health gain and not in promoting health behaviour change as such. Knowledge of the effects on health outcomes, such as the number of cases of disease prevented or years of life gained, makes the beneficial effects of health promotion visible and allows comparison of different interventions. This requires outcome evaluation, which is included in most health education and promotion planning models such as the Precede-Proceed Model ${ }^{1}$. However, in practice this may prove difficult. How, for example, does one determine the health effects of a programme that promotes the consumption of fruits and vegetables?

A major problem with outcome assessment is that most health effects of health behaviour change occur only after many years. Even apart from the delay, this makes measurement of health outcomes complicated and expensive, if not impossible. For example, a decrease in smoking prevalence is followed by a decrease in the incidence of chronic obstructive lung disease several years later, and prevalence and mortality lag even more as incident cases are prevalent for a number of years and then die. Public health professionals and policy-makers are not generally willing to wait that long before deciding whether an intervention is worth the investment.

As an alternative to actual measurement, estimates of health outcomes can be obtained using impact evaluations and epidemiological simulation models. These models are currently used to assess the burden of disease caused by specific risk factors and to estimate the effects of trends in risk factors, but have also been used to assess the health effects of public health interventions ${ }^{2-4}$.

In the present paper we illustrate the use of an epidemiological model and estimate the potential effects on the Dutch population (totalling just over 16 million) of individual nutrition advice via computer-tailored nutrition 
education, a promising behaviour change intervention strategy ${ }^{5}$.

\section{Methods}

The method consists of a two-step process (Fig. 1). The first step is to estimate the effect of an intervention on the exposure of a population to a determinant of health (i.e. the consumption of fruits and vegetables). This is preferably based on a meta-analysis of evaluation studies of a behaviour change intervention approach. If such a meta-analysis is not available, estimates from single wellconducted studies can be used. In the second step an epidemiological model is used to estimate the change in health outcomes due to the intervention-induced change in exposure of a particular population. We shall first introduce the health promotion intervention and its impact evaluation (step 1), then briefly explain the structure and contents of the model (step 2), and discuss how we connected the two steps and what assumptions we made where data were not available.

\section{Computer-tailored nutrition education}

Individualisation of health education interventions via computer-tailoring is regarded as one of the most promising health education techniques, and is effective in inducing dietary behaviour change ${ }^{5}$. In computertailoring, an expert system is used to provide respondents with personally relevant dietary change information based on a personal assessment of dietary intake and potential mediators of change, such as intentions, attitudes and self-efficacy expectations. Brug et al. conducted a pretest-post-test randomised trial of a computer-tailored feedback intervention on fat, fruit and vegetable intakes in Dutch adults ${ }^{6}$. The intervention group received computergenerated feedback letters tailored to their dietary intake, awareness of personal intake levels, intentions, attitudes and self-efficacy expectations. Participants subsequently received a second computer-tailored feedback letter tailored to the changes they made after the initial feedback. A post-test was conducted 4 weeks after this second feedback letter. The reference group received

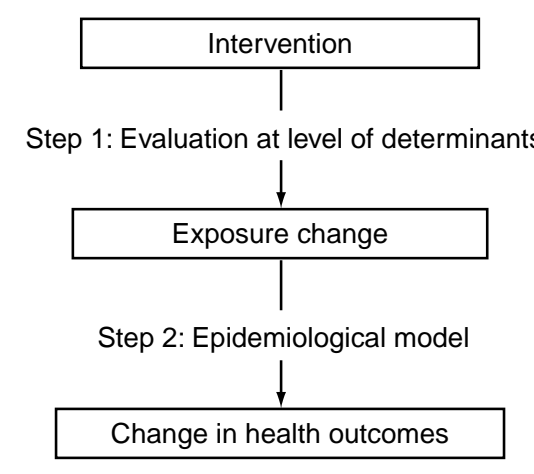

Fig. 1 Overview of methods generic nutrition information. For the present study we compared pre- and post-test measurements of fruit and vegetable consumption and expressed this difference as a percentage of pre-test consumption. The intervention achieved consumption increases of $15 \%$ for fruits and $13.2 \%$ for vegetables.

\section{The fruit and vegetable bealth model}

The effect of a change in fruit and vegetable consumption on health outcomes was estimated using a proportional multi-state life table model $^{7}$. This model compares two populations: one that is modelled after the Dutch population and an identical population for which the fruit and vegetable intake can be manipulated. The mean consumption (in grams per day) is fitted to a Weibull distribution of which the mean is shifted upwards in the intervention population while keeping the shape parameter stable. This results in a new distribution pattern at a higher consumption level. Both curves are translated into discrete consumption categories (see Fig. 2).

Consumption levels influence the incidence of ischaemic heart disease, stroke and cancer of the oesophagus, stomach, colorectum, lung and female breast ${ }^{8,9}$. This effect is quantified via the potential impact fraction (PIF), which is defined as the proportional change in expected incidence as a consequence of a specified change in exposure level. PIF is calculated on the basis of age- and gender-specific exposure data and the relative risks of disease incidence at the corresponding levels of exposure (see Table 1). Changes in the incidence of a disease lead to corresponding changes in prevalence and mortality. Finally, the diseasespecific data of the diseases in the model are integrated in a life table. From this life table, summary measures of population health such as life expectancy, years lost to disease and health-adjusted life-years (DALYs) can be derived $^{10}$. The difference in health outcomes of the two populations is attributed to the intervention, i.e. the change in consumption of fruits and vegetables. The data used to construct the model were the most recent estimates of disease frequency provided by the Dutch National Institute of Public Health and the Environment, population data and mortality rates from the Central Bureau of Statistics,

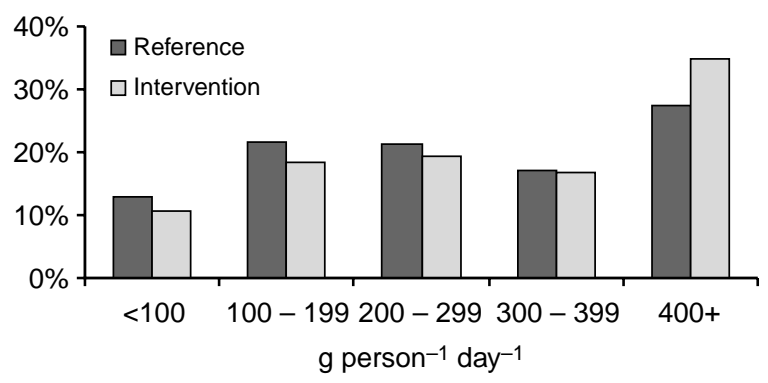

Fig. 2 Example of the estimated change in consumption distribution of fruits and vegetables due to computer-tailored health promotion, assuming participation of the entire population (shown here is the effect on men aged $45-64$ years) 
Table 1 Relative risks of disease incidence for fruit and vegetable consumption

\begin{tabular}{|c|c|c|}
\hline & Relative risk (95\% confidence interval) & Unit \\
\hline Ischaemic heart disease & $0.8(0.65-0.90)^{*}$ & $400+$ versus $0-99 \mathrm{~g} \mathrm{day}^{-1}$ \\
\hline Cerebrovascular accident & $0.8(0.60-0.95)^{*}$ & $400+$ versus $0-99 \mathrm{~g} \mathrm{day}^{-1}$ \\
\hline Oesophagus cancer & $0.81(0.72-0.90)$ & per $100 \mathrm{~g} \mathrm{day}^{-1}$ increase \\
\hline Stomach cancer & $0.78(0.72-0.84)$ & per $100 \mathrm{~g} \mathrm{day}^{-1}$ increase \\
\hline Colorectal cancer & $0.93(0.88-0.98)$ & per $100 \mathrm{~g} \mathrm{day}^{-1}$ increase \\
\hline Lung cancer & $0.87(0.80-0.93)$ & per $100 \mathrm{~g} \mathrm{day}^{-1}$ increase \\
\hline Breast cancer & $0.98(0.96-0.99)$ & per $100 \mathrm{~g} \mathrm{day}^{-1}$ increase \\
\hline
\end{tabular}

${ }^{*}$ For ischaemic heart disease and cerebrovascular accident no confidence intervals were available but the values stated were interpreted as such.

consumption data from the National Food Consumption Survey 1997-1998, estimates of relative risk of coronary heart disease and stroke from a review and of cancer from a recent meta-analysis, and the Dutch disability weights ${ }^{9,11-13}$. The model was implemented in Microsoft ${ }^{\circledR}$ Excel. Ninety-five per cent uncertainty intervals (UI) that express the uncertainty in the protective effects of fruits and vegetables were calculated by Monte Carlo simulation, using the program @ Risk $^{14}$.

\section{Assumptions}

When estimating the effect of an intervention on a population, questions may arise for which an evaluation does not provide direct answers. For this study, we made the following supplementary assumptions. First, the participants in the study by Brug et al. were assumed to be representative of the Dutch adult population. Second, the effect of the intervention was defined as the difference between baseline measurement (T1) and 8 weeks later (T3, 4 weeks after last feedback). The control group was ignored because for this study we were interested in the effect of the intervention versus no intervention, not in the additional effect of tailoring and feedback over generic health education. Third, the results of the intervention were expressed as percentage increase in average total consumption of fruits and vegetables per person per day (as opposed to using absolute numbers of grams, which would have been equally defensible). Fourth, the effects on fruits $(+15.0 \%)$ and vegetables $(+13.2 \%)$ combined are estimated to be the average of the two values (i.e. $+14.1 \%$ ). Finally, changes in consumption are assumed lifelong. This left us with the question what percentage of the Dutch population could be expected to participate in the programme. By way of sensitivity analysis, we calculated outcomes for participation rates of 100\%, 25\% and $10 \%$ of the Dutch population of age 25 years and over.

\section{Results}

The $14.1 \%$ increase in the average consumption of fruits and vegetables results in a shift in the consumption distribution of the Dutch population. As an illustration, Fig. 2 shows the effect on men aged 45-64 years. Assuming a participation rate of $100 \%$, this change in consumption would result in a mortality decrease of about 786 (95\% UI 587-979) deaths annually, or $0.4-0.7 \%$ in relative terms, over 10000 life-years or DALYs (Table 2). Healthy life expectancy would rise by almost 33 (95\% UI 23-43) days for men and 25 (95\% UI 16-34) days for women. When lower participation rates are assumed, health gain diminishes linearly (Table 3 ).

\section{Discussion}

The predicted increase in life expectancy of 3 weeks is about half that of lowering daily intake of salt by $6 \mathrm{~g}$ per person, but reaching that effect would probably require multiple interventions ${ }^{15}$. The Dutch breast cancer screening programme increases female life expectancy by about 5 weeks, but is more demanding in terms of resources and emotions than a computer-tailored fruit and vegetable promotion $^{16}$

The results of this exercise are dependent on the quality of the data and methods used, as well as on a number of assumptions.

\section{Uncertainty in the data}

The results are most sensitive to two kinds of data uncertainties. First, the effect of the intervention was estimated on the basis of a single evaluation, which makes it vulnerable to bias. The use of a formal review of all similar interventions would be preferable, but at present none is available. Second, the estimates of the relative risks of disease for different levels of consumption of fruits and vegetables are subject to considerable uncertainty ${ }^{8,12}$. This reflects the fact that it is not known exactly what the active components in fruits and vegetables are, the difficulty of accurately measuring fruit and vegetable intakes, and controversy over the best study designs to investigate possible preventive effects of fruit and vegetable consumption $^{8}$. Recent cohort studies generally give lower effect estimates than case-control studies; we used estimates based on reviews that included both designs (Table 1) 9,12. $^{2}$.

\section{Uncertainty in the assumptions}

In the analysis a number of assumptions have been made, of which especially the assumed participation rates and 
Table 3 Annual health gain due to computer-tailored health promotion with $10 \%, 25 \%$ and $100 \%$ of the total Dutch population aged 25 years and over participating

\begin{tabular}{lccr}
\hline & \multicolumn{3}{c}{ Participation rate (\%) } \\
\cline { 2 - 4 } & 100 & 25 & 10 \\
\hline Mortality difference & -786 & -196 & -79 \\
Life-years gained & 10547 & 2637 & 1055 \\
DALYs gained & 13789 & 3447 & 1379 \\
Life expectancy at birth (days) & 22.9 & 5.7 & 2.3 \\
DALE at birth ('DA-days') & 29.0 & 7.2 & 2.9
\end{tabular}

DALY - healthy life-years; DALE - healthy life expectancy; DA - disability-adjusted.

the sustainability of the effects influence the health outcomes. In studies on computer-tailored nutrition interventions that have been published, participation rates of up to $80 \%$ have been reported ${ }^{17}$. A tailored intervention targeting highly educated male employees had a participation of $74 \%{ }^{18}$. These rates will be hard to match in the open Dutch population, although much will depend on the recruitment strategy. In our model, participation relates linearly to health outcomes. Because of this assumed linearity, health outcomes can be derived for any assumption regarding participation levels.

The results are also sensitive to assumptions regarding durability of the change in consumption. The effect estimate was obtained in an evaluation 4 weeks after the participants received their last feedback. The results presented are based on the assumption that they continue eating more fruits and vegetables. In one study the effects lasted for at least 8 weeks ${ }^{19}$. Short-term effects of shortterm interventions are, however, often not sustained ${ }^{20}$. Again, different assumptions on sustainability of intervention effects can be used as input in our model. If we assume, for example, that half of the participants fall back to their previous consumption levels soon after the intervention, health effects will also halve. Assessment of fruit and vegetable consumption some years after the intervention could help to determine what longer-term effect can realistically be expected.

Finally, we assumed that the population was representative of the open Dutch population. However, the study population was predominantly female and more highly educated than average, which may have led to overestimation of the effects. There is insufficient evidence to draw firm conclusions about the influence of educational level and gender on the effectiveness of tailored health education. There are indications that it is similarly effective among lower-educated women in the USA ${ }^{21}$, but a study among predominantly highly educated male employees reported only a $5 \%$ increase in fruit and vegetable intake ${ }^{18}$.

\section{Limitations due to the structure of the model}

Apart from the limitations due to the data and uncertainty in the assumptions, the time factor introduces uncertainties that our model does not fully address.
The first is that the model used in this study does not incorporate a time dimension, but instead gives causal effects as immediate. In reality there is a time lag between change in consumption and incidence of disease, and between incidence and mortality. The health gain due to an increase in consumption in a particular year would in reality materialise over a number of subsequent years, but in the end the health gain will be equal to the size of the effect predicted by the model ${ }^{7}$. If it is important to estimate when effects will occur, dynamic models are more appropriate. Dynamic models use the output of one year as input for the next, making the model more realistic but also more complex.

Second, the model uses the present occurrence of disease. As the disease pattern changes with time, so does the effect of the intervention, which means that the accuracy of predictions decreases the further we look into the future. However, patterns of disease in populations generally change slowly, so that accuracy for the first 10 or 20 years will not be biased much.

Finally, this is a macro-simulation model that ignores heterogeneity within the population, such as health differences linked to socio-economic status (SES). Because lower socio-economic groups consume less fruits and vegetables and have higher disease rates, they are likely to gain more health than those with higher SES. In general, micro-simulation models (which have individuals as basic unit) are better suited for the assessment of heterogeneity, but again at the cost of increased complexity and data requirements.

\section{Possibilities for further analysis}

A next step could be to estimate the costs of the intervention programme and perform a cost-effectiveness analysis. This kind of analysis would help decision-makers set priorities and decide whether to invest in a computertailored nutrition advice, a school fruit programme or e.g. a campaign to help smokers quit. For optimal comparability, similar methods should be used for all assessments.

\section{The value offorecasting bealth effects}

Despite the above limitations and the uncertainty inherent in any prediction, epidemiological models can be useful to estimate the effect of interventions on population health.

First, modelling makes the health effects of alternative interventions comparable. This can inform decisions on the allocation of resources for health and aid in setting priorities. Whereas the results of impact evaluations are often difficult to compare, with the help of models different interventions can be translated into forecasts of total health gain in generic terms (e.g. life-years or DALYs) ${ }^{22}$.

Second, epidemiological models provide a logical framework in which evidence can be summarised and assessed for its consequences. This is useful even without actually running the model. As shown above, use of the 
model raises questions that need to be answered in order to estimate health effects of interventions at the population level. How strong is the protective effect of fruits and vegetables? What proportion of the target group will participate? The model splits up the general question 'what is the health effect' into sub-questions. For each of these, evidence needs to be sought and assessed for validity.

This leads to the third advantage of the use of models: it shows where the gaps in the evidence are, and where future research can contribute to reducing uncertainty in effect estimates for interventions.

Fourth, this stepwise approach makes the assessment process transparent. Each of the steps in the thought process can be judged for validity. Alternative assumptions can also be assessed for their consequences.

A fifth advantage is that even when there is little evidence available, at least the possible effects of an intervention can be explored. An uncertainty analysis can be done to establish likely boundaries of potential health effects, and a sensitivity analysis reveals how sensitive the results are to changes in input parameters. In our analysis for example, we included estimates of the health effect assuming a participation rate of $100 \%$. This shows that the health effect of computer-tailored health education is unlikely to yield more than 10000 life-years annually.

In conclusion, health promotion needs to move beyond process evaluation and start assessing its effects on population health. In this venture, epidemiological models can be used to estimate the health effects of health promotion interventions, building upon impact evaluation of these interventions. Since no prediction can be better than the information that it is based upon, thorough evaluation of interventions is crucial. These should preferably be conducted a considerable time after the intervention in order to estimate the durability of the behaviour changes.

\section{Acknowledgements}

We thank Elling Bere (Institute for Nutrition Research, Oslo) for his comments on an earlier version of this article.

The model used in this paper can be obtained from the authors, including explanatory notes and directions on how to adapt the model to different risk factors and populations.

\section{References}

1 Carlson Gielen A, McDonald EM. The precede-proceed planning model. In: Glanz K, Lewis FM, Rimer BK, eds. Health Behavior and Health Education. San Francisco, CA: Jossey-Bass, 1997; 359-83.

2 Ezzati M, Hoorn SV, Rodgers A, Lopez AD, Mathers CD, Murray CJ. Estimates of global and regional potential health gains from reducing multiple major risk factors. Lancet 2003; 362(9380): 271-80.
3 Mooy JM, Gunning-Schepers LJ. Computer-assisted health impact assessment for intersectoral health policy. Health Policy 2001; 57(3): 169-77.

4 Naidoo B, Thorogood M, McPherson K, Gunning-Schepers LJ. Modelling the effects of increased physical activity on coronary heart disease in England and Wales. Journal of Epidemiology and Community Health 1997; 51(2): 144-50.

5 Brug J, Oenema A, Campbell M. Past, present, and future of computer-tailored nutrition education. American Journal of Clinical Nutrition 2003; 77(Suppl. 4): 1028S-34S.

6 Brug J, Glanz K, Van Assema P, Kok G, Van Breukelen GJ. The impact of computer-tailored feedback and iterative feedback on fat, fruit, and vegetable intake. Health Education \& Behavior 1998; 25(4): 517-31.

7 Barendregt JJ, Van Oortmarssen GJ, Van Hout BA, Van den Bosch JM, Bonneux L. Coping with multiple morbidity in a life table. Mathematical Population Studies 1998; 7(1): 29-49, 109.

8 Vianio H, Bianchini F, ed. IARC Handbooks of Cancer Prevention. Vol. 8. Fruit and Vegetables. Lyon: IARC Press, 2003.

9 Jansen MCJF, Van deVijver LPL. Fruits and Vegetables in Chronic Disease Prevention - Another Update. Zoetermeer: Holland Produce Promotion, 2004.

10 Murray CJ, Acharya AK. Understanding DALYs (disabilityadjusted life years). Journal of Health Economics 1997; 16(6): $703-30$.

11 Dutch National Institute of Public Health and the Environment (RIVM). The National Public Health Compass. [online], 2004. Avaliable at http://www.nationaalkompas.nl. Accessed 1 October 2004.

12 Riboli E, Norat T. Epidemiologic evidence of the protective effect of fruit and vegetables on cancer risk. American Journal of Clinical Nutrition 2003; 78(Suppl. 3): 559S-69S.

13 Stouthard MEA, Essink-Bot M-L, Bonsel GJ, Barendregt JJ, Kramers PGN, Van de Water HPA, et al. Disability Weights for Diseases in The Netherlands. Rotterdam: Department of Public Health, Erasmus University Rotterdam, 1997.

14 @RISK 4.0 [computer program]. London: Palisade, 2000.

15 Selmer RM, Kristiansen IS, Haglerod A, Graff-Iversen S, Larsen HK, Meyer HE, et al. Cost and health consequences of reducing the population intake of salt. Journal of Epidemiology and Community Health 2000; 54(9): 697-702.

16 Bonneux L. Mortality reduction by breast-cancer screening. Lancet 2003; 362(9379): 245.

17 Brug J, Campbell M, Van Assema P. The application and impact of computer-generated personalized nutrition education: a review of the literature. Patient Education and Counseling 1999; 36(2): 145-56.

18 Brug J, Steenhuis I, van Assema P, de Vries H. The impact of a computer-tailored nutrition intervention. Preventive Medicine 1996; 25(3): 236-42.

19 Irvine AB, Ary DV, Grove DA, Gilfillan-Morton L. The effectiveness of an interactive multimedia program to influence eating habits. Health Education Research 2004; 19(3): 290-305.

20 De Vries H, Brug J. Computer-tailored interventions motivating people to adopt health promoting behaviors: introduction to a new approach. Patient Education and Counseling 1999; 36(2): 99-105.

21 Campbell MK, Tessaro I, DeVellis B, Benedict S, Kelsey K, Belton L, et al. Effects of a tailored health promotion program for female blue-collar workers: health works for women. Preventive Medicine 2002; 34(3): 313-23.

22 Murray CJ, Ezzati M, Lopez AD, Rodgers A, Vander Hoorn S. Comparative quantification of health risks conceptual framework and methodological issues. Population Health Metrics 2003; 1(1): 1. 